\title{
A cross-disciplinary initiative to analyze past floods
}

\author{
Bruno Wilhelm ${ }^{1}$ and Juan Antonio Ballesteros-Cánovas ${ }^{2}$ \\ 1st Floods Working Group meeting, Grenoble, France, 27-30 June 2016
}

PAGES' Floods Working Group (FWG) contributes to global knowledge of the variability of flood frequency and magnitude. The cross-disciplinary analysis of hydrological extreme events involves different temporal (from seasons to millennia) and spatial scales (regional to continental).

FWG's first workshop, involving 46 researchers from 16 countries (a third being early-career scientists), was the starting point to establish a community devoted to past and present floods and create links between paleoscientists, historians, statisticians, hydrologists and climatologists. The principal objectives were to share disciplinary experiences, promote interdisciplinary collaborations and design future projects and products for the group. Introductory keynote presentations were given before the sessions by N. Macdonald, S. St George, R. Denniston, M. Macklin, B. Valero Garcès, L. Schulte and M. Mudelsee.

The first session reviewed the different archives (i.e. sedimentary, speleothem, tree-ring and historical) and their suitability to document past flood occurrences and magnitude, with a particular focus on their respective advantages and limitations. This unprecedented systematic overview of all types of flood archives motivated participants and generated cross-disciplinary debates. The benefit of gathering flood data provided by different archives and methods was considered as great because of the wealth of information provided in term of data diversity and quantity for further analysis on flood variability.

The second session highlighted the potential for multi-archive flood approaches to get more precise and complete regional flood knowledge, including calibration of flood records with instrumental data. Participants pointed out opportunities and challenges to handle flood archives with different time resolutions. This should be the target of an interdisciplinary group project, with researchers from different fields collaborating towards a common understanding of past flood activity and exploring the possibility to integrate data of a different type within a unique dataset.

The last session was dedicated to statistical and modeling tools that could be applied to flood reconstructions to analyze flood patterns and identify responsible climate forcing. Particular emphasis was placed on the statistical comparison of archive data with instrumental records for flood hazard assessment. In the discussions following this session, the group identified the need to involve more climate modelers, hydrologists and risk managers to improve the understanding of the physical processes controlling the occurrence and magnitude of floods. These scientific collaborations and synergies could help to contribute to the

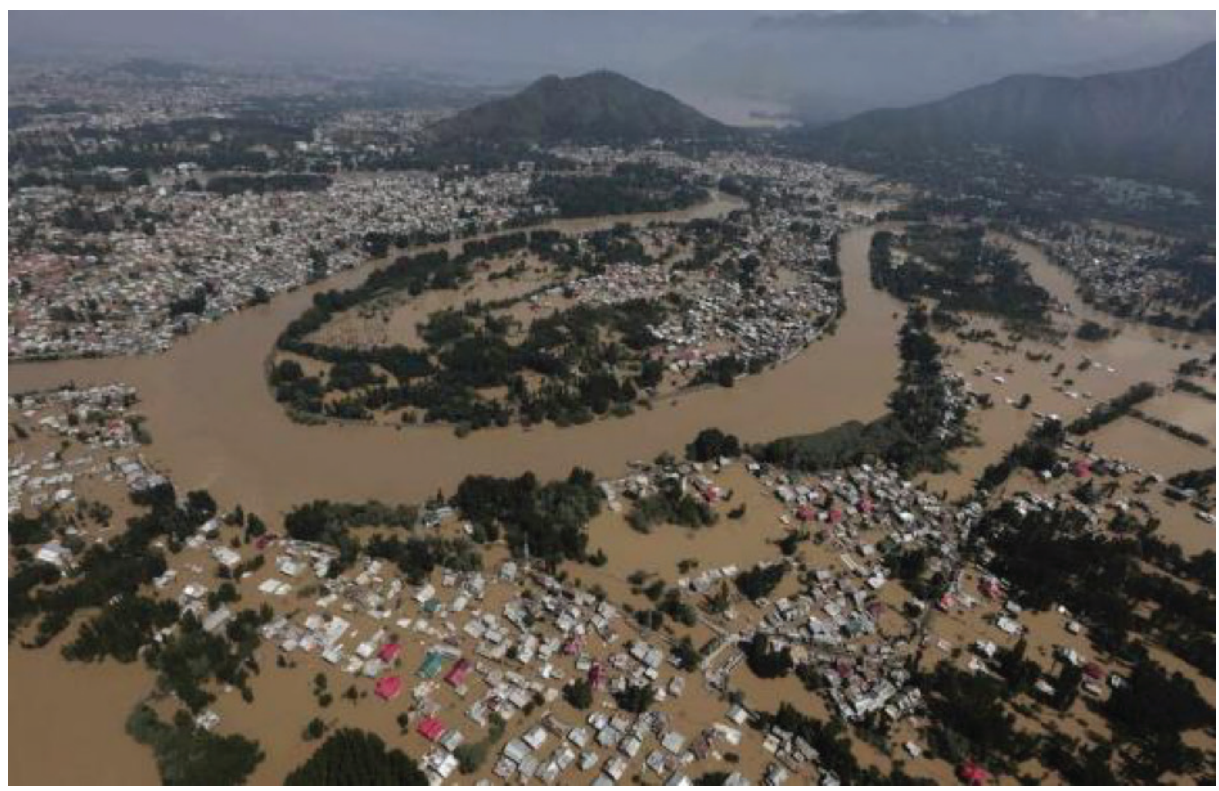

present debate about floods-climate-human linkages and interactions.

Time was also dedicated to sub-group and plenary discussions on three specific topics: (i) the creation of a metadatabase of past flood events, (ii) the need to communicate the archive-based flood data to other communities, and (iii) collaborative efforts within the FWG. Based on a consensus, FWG's next steps will be the creation of this metadatabase, gathering published data, followed by its analysis in a review paper. The metadata collection will be done by groups, each focusing on a specific type of archive. First, each group will identify the archive type-specific selection criteria for metadata collection and a set of common criteria will then be defined. Once this first database version is finalized, a format for the inclusion of the actual data will be defined and the data collection planned. The format for the database will build upon the work of other PAGES working groups (e.g. McKay and Emile-Geay 2016). Deadlines for these three steps were established in order to deliver the first metadatabase version at the PAGES OSM in Zaragoza (9-13 May 2017), where the next FWG meeting and the FWG-sponsored session "Multidisciplinary reconstruction of paleofloods" will take place.

Post-workshop materials (report, keynotes, talks and posters) are available here:

www.pastglobalchanges.org/calendar/127-pages/1553-cross-community-workshop-on-past-flood-variability

\section{ACKNOWLEDGEMENTS}

The workshop has been generously supported by PAGES, Labex OSUG@2020 (Investissements d'avenir - ANR10 LABX56), European Geosciences Union, Grenoble-INP and Université Grenoble Alpes.

\section{AFFILIATIONS}

'LTHE, University Grenoble Alpes (CNRS, IRD), Grenoble, France ${ }^{2}$ Environmental Science, University of Geneva, Switzerland

\section{CONTACT}

Name: bruno.wilhelm@ujf-grenoble.fr

\section{REFERENCE}

McKay NP, Emile-Geay J (2016) Clim Past 12: 1093-1100

Figure 1: September 2014 flood event in Kashmir valley (India) leading to the complete submersion of houses of the largest city, Srinagar, during several days (National Disaster Response Force - NDRF). 